\title{
Time Truncated Chain Sampling Plans for Marshall-Olkin Extended Exponential Distributions
}

\author{
Dr. A. R. Sudamani Ramaswamy ${ }^{1,}$ S.Jayasri $^{2}$ \\ 1. Associate Professor, Department of Mathematics, Avinashilingam University, Coimbatore. \\ 2 Assistant professor, Department of Mathematics, CIT, Coimbatore.
}

\begin{abstract}
Chain sampling plan for marshall olkin extended exponential distribution when the life-test is truncated at a pre-specified time are provided in this manuscript. The design parameters such as the minimum sample size and the acceptance number are obtained by satisfying the producer's and consumer's risks at the specified quality levels, under the assumption that the termination time and the number of items are pre-fixed.

Keywords - Truncated life test, Marshall olkin extended exponential distribution, Operating characteristics, Consumer's risk, Producer's risk.
\end{abstract}

\section{Introduction:}

An acceptance sampling plan involves quality contracting on product orders between the producers and the consumers. It is an essential tool in the Statistical Quality Control. In most of the statistical quality control experiment, it is not possible to perform hundred percent inspection, due to various reasons. The acceptance sampling plan was applied in the US Military for testing the bullets during World War II. For example, if every bullet was tested in advance, no bullets were available for shipment, and on the other hand if no bullets were tested, then disaster might occur in the battle field at the crucial time.

In most acceptance sampling plans for a truncated life test, major issue is to determine the sample size from the lot under consideration. It is implicitly assumed in the usual sampling plan that only a single item is put in tester. Sampling inspection in which the criteria for acceptance and non acceptance of the lot depend in part on the results of the inspection of immediately preceding lots is adopted in Chain Sampling Plan. These lifetests are discussed by many authors, Goode and Kao(1961), Gupta and Groll(1961), Baklizi and EI Masri(2004), Rosaiah and Kantam (2005) and Tsai, Tzong and Shou(2006), Balakrishnan, Victor Leiva \& Lopez (2007). All these authors developed the sampling plans for life tests using single acceptance sampling.

The purpose of this study is to find the probability of Acceptance for chain sampling plan assuming the experiment is truncated at preassigned time and lifetime follows a Marshall olkin extended exponential distribution. Suppose $\mathrm{n}$ units are placed in a life test and the experiment is stopped at a Predetermined time T. The number of failures till the time point $\mathrm{T}$ is observed, and suppose it is $\mathrm{d}$. The decision to accept the lot takes place, if and only if the number of failures $d$ at the end of the time point $T$ does not exceed the specified acceptance number .

\section{GLOSSARY OF SYMBOLS:}

$\begin{array}{lll}\mathrm{n} & - & \text { Sample size } \\ \alpha & - & \text { Index parameter } \\ \sigma & - & \text { Scale parameter } \\ \mathrm{T} & - & \text { Prefixed time } \\ \mathrm{p}^{*} & - & \text { Minimum probability } \\ \beta & - & \text { Consumer's risk } \\ \mathrm{d} & - & \text { Number of defectives } \\ \mathrm{p} & - & \text { Failure probability } \\ \mathrm{L}(\mathrm{p})- & \text { Probability of acceptance } \\ \mathrm{i} \quad- & \text { Acceptance criteria }\end{array}$

III. Marshall-Olkin Extended Exponential Distributions :

Marshall and olkin (1997) introduced an interesting method of adding a new parameter to an existing distribution. The resulting new distribution, known as the Marshall-olkin extended distribution. The probability density function (p. d. f.) and cumulative distribution function (c. d. f.) of the Marshall olkin extended exponential distribution are given by 


$$
g(x ; \alpha, \sigma)=\frac{\frac{\alpha}{\lambda} e^{-\frac{t}{\sigma}}}{\left(1-\bar{\alpha}^{-\frac{t}{\sigma}}\right)^{2}} ; \quad \mathrm{t}>0, \quad \alpha, \sigma>0, \quad \bar{\alpha}=1-\alpha
$$

And

$$
G(x ; \alpha, \sigma)=\frac{1-e^{-\frac{t}{\sigma}}}{\left(1-\bar{\alpha} e^{-\frac{t}{\sigma}}\right)} ; \quad \mathrm{t}>0, \quad \alpha, \sigma>0, \quad \bar{\alpha}=1-\alpha
$$

$\alpha$ is the index parameter and let us assume $\alpha=2, \sigma$ is the scale parameter. If some other parameters are involved, then they are assumed to be known, for example, if shape parameter of a distribution is unknown it is very difficult to design the acceptance sampling plan. In quality control analysis, the scale parameter is often called the quality parameter or characteristics parameter. Therefore it is assumed that the distribution function depends on time only through the ratio of $t / \sigma$.

\section{Chain Sampling Plan :}

Chain Sampling Plan (ChSP-1) proposed by Dodge (1955) making use of cumulative results of several samples help to overcome the shortcomings of the Single Sampling Plan. It avoids rejection of a lot on the basis of a single nonconfirming unit and improves the poor discrimination between good and bad quality that occurs with the $\mathrm{c}=0$ plan.

The use of cumulative results of several samples is proposed for application to cases where there is repetitive production under the same conditions and where the lots or batches of products to be inspected are submitted for inspection in the order of production. Such situation may arise in receiving inspection of a continuing supply of purchased materials produced with in a manufacturing plan. Chain sampling is not suited to intermittent or job lot production, or to occasional purchases. An example situation is a continuing supply of processed material, such as a particular type of copper alloy rod

When large samples are not practicable, and the use of $c=0$ plan is warrented, for example, when an extremely high quality is essential the use of chain sampling plan is often recommended. The conditions for application and operating procedure of chsp- 1 are as follows

\subsection{Conditions for application of ChSP -1:}

The cost of destructiveness of testing is such that a relatively small sample size is necessary, although other factors make a large sample desirable.

1) The product to be inspected comprises a series of successive lots produced by a continuing process.

2) Normally lots are expected to be of essentially the same quality.

3) The consumer has faith in the integrity of the producer.

\subsection{Operating Procedure:}

The plan is implemented in the following way:

1) For each lot, select a sample of $\mathrm{n}$ units and test each unit for conformance to the specified requirements.

2) Accept the lot if $d$ (the observed number of defectives) is zero in the sample of $n$ units, and reject if $d>1$.

3) Accept the lot if $d$ is equal to 1 and if no defectives are found in the immediately preceeding i samples of size n.

Dodge (1955) has given the operating characteristic function of ChSP-1 as $\mathrm{P}_{\mathrm{a}}(\mathrm{p})=\mathrm{P}_{0}+\mathrm{P}_{1}\left(\mathrm{P}_{0}\right)^{\mathrm{i}}$,

Where $P_{a}=$ the probability of acceptance,

$\mathrm{P}_{0}=$ probability of finding no defects in a sample of $\mathrm{n}$ units from product of quality $\mathrm{p}$.

$\mathrm{P}_{1}=$ probability of finding one defect in such a sample.

$\mathrm{i}=$ Number of preceding samples.

The Chain sampling Plan is characterized by the parameters $n$ and i. We are interested in determining the sample size required for in the case of marshall -olkin extended exponential distribution and various values of acceptance number i.

The probability $(\alpha)$ of rejecting a good lot is called the producer's risk, whereas the probability $(\beta)$ of accepting a bad lot is known as the consumer's risk. Often the consumer risk is expressed by the consumer's confidence level. If the confidence level is $p^{*}$ then the consumer's risk will be $\beta=1-p^{*}$.We will determine the sample size so that the consumer's risk does not exceed a given value $\beta$.

The probability of acceptance can be regarded as a function of the deviation of specified average from the true average. This function is called operating characteristic (oc) function sampling plan. Once 
the minimum sample size is obtained one may be interested to find the probability of acceptance of a lot when the quality of the product is good enough. The probability of acceptance in the case of chain sampling plan is calculated using the following equation given by

$$
\begin{aligned}
& \text { Where } \quad \mathrm{P}=\frac{1-e^{-\frac{t}{\sigma}}(\mathrm{p})=(1-\mathrm{p})^{n}+\mathrm{np}(1-\mathrm{p})^{n-1}(1-\mathrm{p})^{n i}}{\left(1-\bar{\alpha} e^{-\frac{t}{\sigma}}\right)}
\end{aligned}
$$

It is clear that $\mathrm{p}$ depends only on the ratio $t / \sigma_{0}$ for a fixed $\alpha=\alpha_{0}$

In Table 1 we present the minimum values of $n$, satisfying equation for $\mathrm{p}^{*}=0.75,0.90,0.95,0.99$ and for $\mathrm{t} / \sigma_{0}$ $0.628,0.942,1.257,1.571,2.356,3.141,4.712$, keeping $\alpha_{0}$ fixed. These choices are consistent with Gupta and Groll (1961), Gupta (1962), Kantam et al (2001), Baklizi and EI Masri (2004), Balakrishnan et Al (2007).

\section{Description Of Tables And An Example:}

Assume that the life time distribution is an Marshall olkin extended exponential distribution with $\alpha_{0}=2$ and that the experimenter is interested in knowing that the true median life is atleast 1000 hours with confidence 0.99. It is assumed that the maximum affordable time is 767 hours and $t / \sigma_{0}=0.942$, From the table 1 , we obtain $\mathrm{n}=10$. Therefore, out of 10 items if not more than 1 item fail and if no defectives are found in the immediately preceeding i samples before $\mathrm{T}=767$ units of time, the lot can be accepted with the assurance that the true median life is atleast 1000 with probability 0.99 .

For the sampling plan $\left(\mathrm{n}=10, \mathrm{i}=2, \mathrm{t} / \sigma_{0}=0.942\right)$ and confidence level $\mathrm{p}^{*}=0.99$ under an Marshall olkin extended exponential distribution with $\alpha_{0}=2$ the values of the operating characteristic function from

Table 2 as follows

\begin{tabular}{|l|l|l|l|l|l|l|}
\hline$\sigma / \sigma_{0}$ & 2 & 4 & 6 & 8 & 10 & 12 \\
\hline $\mathrm{L}(\mathrm{p})$ & 0.073222 & 0.319000 & 0.515837 & 0.646907 & 0.733780 & 0.793094 \\
\hline
\end{tabular}

\begin{tabular}{|c|c|c|c|c|c|c|c|c|c|}
\hline \multirow[t]{2}{*}{$\mathrm{p}^{*}$} & \multirow[t]{2}{*}{$\mathrm{i}$} & \multicolumn{8}{|l|}{$t / \sigma$} \\
\hline & & 0.628 & 0.942 & 1.257 & 1.571 & 2.356 & 3.141 & 3.927 & 4.712 \\
\hline \multirow[t]{6}{*}{0.75} & 1 & 7 & 4 & 3 & 2 & 2 & 1 & 1 & 1 \\
\hline & 2 & 6 & 4 & 3 & 2 & 1 & 1 & 1 & 1 \\
\hline & 3 & 6 & 4 & 2 & 2 & 1 & 1 & 1 & 1 \\
\hline & 4 & 6 & 3 & 2 & 2 & 1 & 1 & 1 & 1 \\
\hline & 5 & 6 & 3 & 2 & 2 & 1 & 1 & 1 & 1 \\
\hline & 6 & 6 & 3 & 2 & 2 & 1 & 1 & 1 & 1 \\
\hline \multirow[t]{6}{*}{0.90} & 1 & 11 & 6 & 4 & 3 & 2 & 2 & 1 & 1 \\
\hline & 2 & 10 & 6 & 4 & 3 & 2 & 1 & 1 & 1 \\
\hline & 3 & 10 & 5 & 4 & 3 & 2 & 1 & 1 & 1 \\
\hline & 4 & 10 & 5 & 4 & 3 & 2 & 1 & 1 & 1 \\
\hline & 5 & 10 & 5 & 4 & 3 & 2 & 1 & 1 & 1 \\
\hline & 6 & 10 & 5 & 4 & 3 & 2 & 1 & 1 & 1 \\
\hline \multirow[t]{6}{*}{0.95} & 1 & 13 & 7 & 5 & 4 & 2 & 2 & 2 & 1 \\
\hline & 2 & 13 & 7 & 5 & 4 & 2 & 2 & 1 & 1 \\
\hline & 3 & 13 & 7 & 5 & 4 & 2 & 2 & 1 & 1 \\
\hline & 4 & 13 & 7 & 5 & 4 & 2 & 2 & 1 & 1 \\
\hline & 5 & 13 & 7 & 5 & 4 & 2 & 2 & 1 & 1 \\
\hline & 6 & 13 & 7 & 5 & 4 & 2 & 2 & 1 & 1 \\
\hline \multirow{6}{*}{0.99} & 1 & 19 & 11 & 7 & 5 & 3 & 2 & 2 & 2 \\
\hline & 2 & 19 & 10 & 7 & 5 & 3 & 2 & 2 & 2 \\
\hline & 3 & 19 & 10 & 7 & 5 & 3 & 2 & 2 & 2 \\
\hline & 4 & 19 & 10 & 7 & 5 & 3 & 2 & 2 & 2 \\
\hline & 5 & 19 & 10 & 7 & 5 & 3 & 2 & 2 & 2 \\
\hline & 6 & 19 & 10 & 7 & 5 & 3 & 2 & 2 & 2 \\
\hline
\end{tabular}

Table 1:

Minimum sample size for the specified ratio $t / \sigma_{0}$, confidence level $\mathrm{p}^{*}$, and the corresponding acceptance number $i$ when the shape parameter $\alpha=2$ 
Time truncated chain sampling plans for marshall olkin extended exponential distributions

Table 2:

OC values for the time truncated chain sampling plan $\left(n, i, t / \sigma_{0}\right)$ for a given $p^{*}$, when $i=2$ and $\alpha=2$.

\begin{tabular}{|c|c|c|c|c|c|c|c|c|}
\hline \multirow[t]{2}{*}{$\mathrm{p}^{*}$} & \multirow[t]{2}{*}{$\mathrm{n}$} & \multirow{2}{*}{$t / \sigma_{0}$} & \multicolumn{6}{|l|}{$\sigma / \sigma_{n}$} \\
\hline & & & 2 & 4 & 6 & 8 & 10 & 12 \\
\hline \multirow[t]{8}{*}{0.75} & 6 & 0.628 & 0.414740 & 0.730399 & 0.850451 & 0.905739 & 0.935352 & 0.952970 \\
\hline & 4 & 0.942 & 0.400535 & 0.726316 & 0.849318 & 0.905489 & 0.935396 & 0.953115 \\
\hline & 3 & 1.257 & 0.386816 & 0.722101 & 0.848072 & 0.905154 & 0.935377 & 0.953211 \\
\hline & 2 & 1.571 & 0.464227 & 0.781810 & 0.886231 & 0.930791 & 0.953604 & 0.966779 \\
\hline & 1 & 2.356 & 0.588129 & 0.859558 & 0.932130 & 0.960379 & 0.974113 & 0.981787 \\
\hline & 1 & 3.141 & 0.422015 & 0.772969 & 0.885762 & 0.932143 & 0.955250 & 0.968335 \\
\hline & 1 & 3.927 & 0.291862 & 0.680254 & 0.831797 & 0.898131 & 0.932119 & 0.951661 \\
\hline & 1 & 4.712 & 0.197978 & 0.588129 & 0.772931 & 0.859558 & 0.905348 & 0.932130 \\
\hline \multirow[t]{8}{*}{0.90} & 10 & 0.628 & 0.195507 & 0.515837 & 0.694648 & 0.793094 & 0.851410 & 0.888415 \\
\hline & 6 & 0.942 & 0.222288 & 0.557782 & 0.730399 & 0.821066 & 0.873253 & 0.965739 \\
\hline & 4 & 1.257 & 0.256748 & 0.604490 & 0.767634 & 0.849131 & 0.894655 & 0.922440 \\
\hline & 3 & 1.571 & 0.272166 & 0.627536 & 0.786077 & 0.862983 & 0.905179 & 0.930627 \\
\hline & 2 & 2.356 & 0.246162 & 0.615450 & 0.781880 & 0.861648 & 0.904935 & 0.930817 \\
\hline & 1 & 3.141 & 0.422015 & 0.772969 & 0.885762 & 0.932143 & 0.955250 & 0.968335 \\
\hline & 1 & 3.927 & 0.291862 & 0.680254 & 0.831797 & 0.898131 & 0.932119 & 0.951661 \\
\hline & 1 & 4.712 & 0.197978 & 0.588129 & 0.772931 & 0.859558 & 0.905348 & 0.932130 \\
\hline \multirow[t]{8}{*}{0.95} & 13 & 0.628 & 0.113997 & 0.392158 & 0.585838 & 0.705821 & 0.782049 & 0.832689 \\
\hline & 7 & 0.942 & 0.167097 & 0.485634 & 0.672210 & 0.776750 & 0.839200 & 0.879018 \\
\hline & 5 & 1.257 & 0.172439 & 0.501366 & 0.687817 & 0.789819 & 0.849803 & 0.887636 \\
\hline & 4 & 1.571 & 0.162523 & 0.494696 & 0.684730 & 0.788410 & 0.849168 & 0.887376 \\
\hline & 2 & 2.356 & 0.246162 & 0.615450 & 0.781880 & 0.861648 & 0.904935 & 0.930817 \\
\hline & 2 & 3.141 & 0.124880 & 0.464403 & 0.670377 & 0.781915 & 0.846325 & 0.886292 \\
\hline & 1 & 3.927 & 0.291862 & 0.680254 & 0.831797 & 0.898131 & 0.932119 & 0.951661 \\
\hline & 1 & 4.712 & 0.197978 & 0.588129 & 0.779310 & 0.859558 & 0.905348 & 0.932130 \\
\hline \multirow[t]{8}{*}{0.99} & 19 & 0.628 & 0.040333 & 0.227694 & 0.409572 & 0.546749 & 0.645522 & 0.716948 \\
\hline & 10 & 0.942 & 0.073222 & 0.319000 & 0.515837 & 0.646907 & 0.733780 & 0.793094 \\
\hline & 7 & 1.257 & 0.080381 & 0.342295 & 0.542865 & 0.671884 & 0.755397 & 0.811474 \\
\hline & 5 & 1.571 & 0.099010 & 0.387818 & 0.590297 & 0.713213 & 0.789868 & 0.840052 \\
\hline & 3 & 2.356 & 0.108203 & 0.421411 & 0.627634 & 0.746267 & 0.817560 & 0.863030 \\
\hline & 2 & 3.141 & 0.124880 & 0.464403 & 0.670377 & 0.781915 & 0.846325 & 0.886292 \\
\hline & 2 & 3.927 & 0.061967 & 0.340904 & 0.562395 & 0.698171 & 0.781852 & 0.835842 \\
\hline & 2 & 4.712 & 0.030249 & 0.246162 & 0.464344 & 0.615450 & 0.714998 & 0.781880 \\
\hline
\end{tabular}

FIGURE 1 :

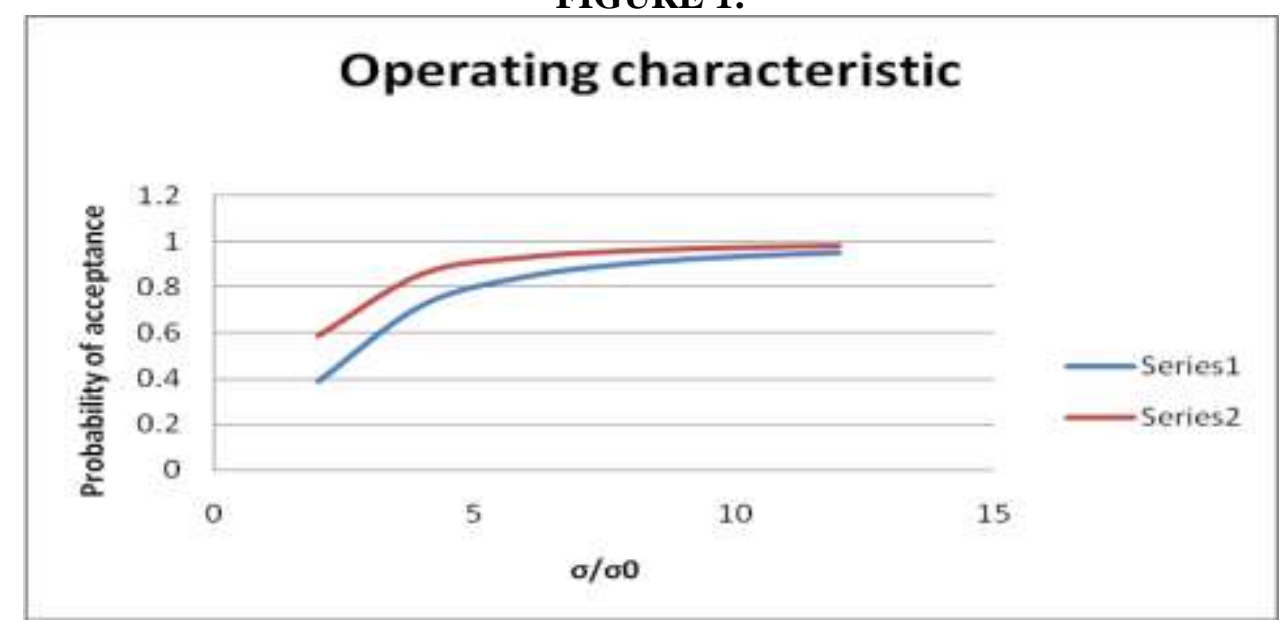

OC curve for Probability of acceptance against $\square / \square_{0}$ 


\section{Conclusions:}

In this paper, chain sampling plan for the truncated life test was proposed in the case of Marshall olkin extended exponential distribution. It is assumed that the shape parameter is known, the minimum sample size required and the acceptance number were calculated. From the figure 1 we can see that the probability of acceptance increases when $\sigma / \sigma_{0}$ increases and it reaches the maximum value 1 when $\sigma / \sigma_{0}$ is greater than 8

\section{References:}

[1] Baklizi,a. and El Masri,A.E.K. (2004), "Acceptance sampling plans based on truncated life tests in the Birnbaum Saunders model", Risk Analysis, vol.24,1453-1457.

[2] Baklizi,a. (2003) "Acceptance sampling plans based on truncated life tests in the Pareto distribution of second kind ", Advances and Applications in Statistics, vol.3,33-48

[3] Balakrishnan, N., Leiva,V. and Lopez, J. (2007), "Acceptance sampling plans from truncated life tests based on generalized Birnbaum Saunders distribution", communications in statistics - simulation and computation, vol.36,643-656.

[4] Dodge,H.F.(1955): Chain Sampling Plan.

[5] Gupta,S.S. and Groll,P.A. (1961), "Gamma distribution in acceptance sampling based on life tests",Journal of the American Statistical Association, vol.56,942-970.

[6] Srinivasa Rao (2010), "Group acceptance sampling plans based on truncated life tests for Marshall - olkin extended Lomax distribution",Electronic journal of Applied Statistical Analysis, Vol.3,Isse 1 (2010),18-27.

[7] Srinivasa Rao (2010), "Double acceptance sampling plans based on truncated life tests for Marshall - olkin extended exponential distribution”, Austrian journal of Statistics, Vol.40, (2011),Number 3, 169-176.

[8] Srinivasa Rao (2009), "Reliability test plans for Marshall - olkin extended exponential distribution", Applied Mathematical Sciences , Vol.3, (2009),Number 55, 2745-2755. 